\title{
IMPACT OF CERTAIN EDUCATIONAL INTERVENTIONS ON ADVERSE DRUG REACTION REPORTING BY NURSING HEALTH PROFESSIONALS AT A TERTIARY CARE HOSPITAL
}

\author{
SUPRIYA SONOWAL ${ }^{1 *}$, CHETNA K DESAI ${ }^{2}$, JIGAR R PANCHAL ${ }^{2}$ \\ ${ }^{1}$ Department of Clinical Science, Sun Pharma Advanced Research Company, Mumbai, Maharashtra, India. ${ }^{2}$ Department of Pharmacology, \\ B. J. Medical College, Ahmedabad, Gujarat, India. Email: supriyasonowal@gmail.com
}

Received: 25 February 2020, Revised and Accepted: 20 April 2020

\section{ABSTRACT}

Objectives: The objectives of the study were to evaluate the impact of certain educational interventions on adverse drug reaction (ADR) reporting by nursing health professionals at a tertiary care hospital.

Methods: Knowledge, attitude, and practice (KAP) of the nurses regarding ADR reporting were evaluated before and after interventions using a KAP questionnaire. Educational interventions carried out among the nurses were workshops, booklets, SMSes, personal briefings, and posters. Number and quality of ADR reported by nurses in pre-intervention (3 months), intervention (10 months), and post-intervention ( 3 months) phase were compared.

Results: There was a significant increase in response rate to questionnaires in the post-intervention phase (post-IP) (97.74\%) as compared to preintervention (91.28\%) phase (pre-IP). The knowledge score of the nurses increased significantly in post-IP (11.65 \pm 2.14$)$ as compared to the preintervention (6.98 \pm 2.46$)$ phase. No ADR was reported by nurses in pre-IP. Thirty nurses reported 30 ADRs in the intervention phase and six nurses reported six ADRs in the post-IP. The mean score of completeness of ADR notification forms decreased significantly in post-IP. Maximum ADRs (10) were reported after the workshops.

Conclusion: Educational interventions improved the KAP of ADR reporting by nurses, albeit temporary. This suggests the need of continuous educational interventions.

Keywords: Adverse drug reaction, Educational interventions, Nurses, Adverse drug reaction reporting.

(C) 2020 The Authors. Published by Innovare Academic Sciences Pvt Ltd. This is an open access article under the CC BY license (http://creativecommons. org/licenses/by/4. 0/) DOI: http://dx.doi.org/10.22159/ajpcr.2020.v13i6.37266

\section{INTRODUCTION}

No medicine is absolutely safe. Safe use of medicines is a major challenge for health-care professionals. Studies conducted worldwide have proved the burden of adverse drug reactions (ADRs). ADRs are one of the leading causes of mortality, morbidity, hospital admissions, and increased expenditure for health-care industry [1-4]. Hence, safety monitoring of drugs is essential for better patient care. In this context, pharmacovigilance has a promising role. Pharmacovigilance has gained momentum in India following the initiation of Pharmacovigilance Programme of India (PvPI)."

Underreporting ofADRsisamajorchallengetopharmacovigilance.Studies have shown that only $6-10 \%$ of ADRs are reported spontaneously [5]. Participation of all categories of health-care professionals is one of the key factors for the success of a pharmacovigilance program [6]. PvPI encourages all health-care professionals to report ADRs. Doctors are the major contributors to ADRs reporting currently [7]. ADR reporting by other health professionals is minimal in India, albeit initiated in some countries. Lack of awareness of the importance and need for pharmacovigilance and ADR reporting exists. Hence, interventions to sensitize other health professionals are required to widen the scope of pharmacovigilance in India.

Nurses are the health professionals who are responsible for taking care of patients round the clock. Hence, a possibility exists that nurses may notice an ADR earlier than doctors do. Given their basic medical knowledge and constant association with patients, nurses can detect ADRs which may go unnoticed otherwise. Educational interventions such as continuing medical educations, workshops, periodic meetings on pharmacovigilance, short message services (SMS), e-mails, face to face briefings, economic incentives, telephone calls, and posters have proven to improve ADR reporting across the globe [8-11]. Educational interventions have shown evidence of improved ADR reporting by prescribers at Civil Hospital Ahmedabad (CHA) [11]. It is therefore hypothesized that similar educational interventions in nurses may help initiate and improve the practice of ADR reporting by this vital pool of health-care provider. Hence, this study was designed to evaluate the knowledge, attitude, and practice (KAP) of ADR reporting and to evaluate the impact of educational interventions on ADR reporting by the nursing health professionals of CHA.

\section{MATERIALS AND METHODS}

This was a continuous, prospective, open, comparative, interventional, single-center study carried out for 16 months at Civil Hospital, Ahmedabad, a 2200 bedded tertiary care hospital in Gujarat, India. Prior permission was sought from the Institutional Ethics Committee of Civil Hospital, Ahmedabad and from the Medical Superintendent of CHA to display posters in the wards and organize workshops for nurses. A sample of 195 nurses was selected from a total of 824 nurses working in the hospital. The nurses working in medicine (6), surgery (4), obstetrics and gynecology (4), pediatrics (2), psychiatry, and dermatology wards were included in the study. Nurses not willing to consent and nurses posted on a temporary basis were not included in the study. The study population comprised 36 head nurses and 159 staff nurses.

The study was carried out in three phases:

Pre-interventional phase (pre-IP)

The KAP of the nurses was evaluated through a pre-validated KAP questionnaire. The questionnaire was pretested and validated in 20 randomly selected nurses. Questionnaire was suitably modified 
and also translated to Gujarati by a certified language expert. After written informed consent, the questionnaire was administered to the study population $(\mathrm{n}=195)$. A notification form consisting of essential information required to notify ADRs was prepared and validated in English and Gujarati. The "ADR diary," comprised of ADRs of drugs commonly used, was also prepared in English and Gujarati.

\section{Interventional phase (IP)}

The IP comprised various interventions aimed to initiate ADR reporting, i.e. workshops, ADR drop boxes, SMSes, posters, ADR diary, and personal briefings. At the start of this phase, five workshops on pharmacovigilance and importance of ADR reporting were conducted to train all participating nurses about the importance and method of ADR reporting. Nurses were also briefed about the study procedures during workshops. Hands-on exercises on ADR reporting and notification using CDSCO ADR reporting form and notification form was also included in the workshop. The "ADR diary" was distributed to the nurses at the workshops. ADR reporting forms and notification forms were distributed in the wards. ADR drop boxes were prepared and placed at the nursing station of each ward. SMSes that reminded nurses to report ADRs were sent fortnightly to all the nurses. The importance of ADR reporting, role of nurses in ADR reporting and feedback of ADRs reported by them were included in these SMSes. Posters emphasizing pharmacovigilance and importance of ADR reporting were displayed at the nursing stations of each ward, the hospital corridors, and other public places of CHA and were changed once a month. The investigator met each nurse once a month. In the personal briefings besides urging the nurses to report ADRs, the importance and role of nurses in ADR reporting were reiterated. Queries, suggestions, feedback, and problems faced in reporting ADRs, if any, were also addressed.

\section{Post-interventional phase (post-IP)}

During this phase, the nurses were re-evaluated for their KAP of ADR reporting using the KAP questionnaire. Spontaneous reporting of ADRs by the nurses was also evaluated.

\section{Data analysis}

Questions to evaluate o knowledge about ADRs were scored on a scale of $0-15$. The scores obtained in pre-IP and post-IP were compared using the t-test. Questions to test the ADR reporting, attitude, and practice were evaluated for percentage responses and compared between preIP and post-IP using the Chi-square test. The number of ADRs reported in each phase was recorded and their relationship with interventions was evaluated. The number of nurses who reported ADRs in each phase was compared between the phases using the Chi-square test. ADRs were also evaluated for the method of their reporting, causal drug, symptoms, causality, preventability, and severity. The completeness of ADR notification forms was evaluated on a scale of $0-30$.

\section{RESULTS}

A total of 195 nurses were enrolled in this cross-sectional, interventional, and prospective study carried out among the nursing health professionals of CHA. Three nurses had retired and 15 nurses were transferred to other places during the study period. Hence, 177 nurses completed the study. The mean age of the nurses was $(36.57 \pm 10.42)$ years. All the nurses, except one, were females (male: female - 1:194). The study population comprised 159 (81.5\%) staff nurses and $36(18.5 \%)$ head nurses.

The response rate to KAP questionnaire was $91.28 \%$ in pre-IP and $97.74 \%$ in post-IP, indicating a significant increase in the post-IP $(\mathrm{P}<0.05)$.

\section{Knowledge}

The maximum score allotted to the seven questions that evaluate the knowledge about ADR was 15. A significant increase was observed in the mean knowledge score in the post-IP $(11.65 \pm 2.14)$ as compared to pre-IP $(6.98 \pm 2.46)(\mathrm{P}<0.001)$. The number of nurses who gained knowledge about pharmacovigilance and ADR reporting increased significantly in post-IP (Table 1).

\section{Attitude}

Four of 14 questions assessed the attitude of nurses toward ADR reporting. A significantly higher number of nurses in the Post-IP $(159 / 173,91.91 \%)$ opined that nurses should report ADRs than in preIP $(140 / 178,78.65 \%)(P<0.001)$. A significantly greater number of nurses in the Post-IP (129/173) strongly agreed that it is important to report ADRs than those in the pre-IP $(99 / 178)(\mathrm{P}<0.001)$. Similarly,

Table 1: Knowledge attitude and practice of nurses at CHA toward ADR reporting in pre-IP and post-IP

\begin{tabular}{|c|c|c|}
\hline Assessment of knowledge & Pre-IP, n (\%) & Post-IP, n (\%) \\
\hline Are you aware of the word Pharmacovigilance? & $113(63.48 \%)$ & $168(97.11 \%)^{* *}$ \\
\hline Do you know the meaning of "Pharmacovigilance"? & $105(58.99 \%)$ & $156(90.17 \%)^{* *}$ \\
\hline \multicolumn{3}{|l|}{ Three common medicines that cause ADRs } \\
\hline Antimicrobial & $82(46.06 \%)$ & $160(92.49 \%)^{* *}$ \\
\hline Analgesic & $69(38.76 \%)$ & $91(52.6 \%)^{* *}$ \\
\hline Perinorm & $64(35.96 \%)$ & $62(35.84 \%)$ \\
\hline \multicolumn{3}{|l|}{ Three common ADRs } \\
\hline Gastritis & $60(33.71 \%)$ & $98(56.65 \%)^{* *}$ \\
\hline Involuntary movement & $62(29.21 \%)$ & $54(31.21 \%)$ \\
\hline Are you aware of drugs banned due to ADRs? & $80(44.94 \%)$ & $126(72.83 \%)^{* *}$ \\
\hline Are you aware of ADR reporting system of CHA? & $66(37.08 \%)$ & $154(89.02 \%)^{* *}$ \\
\hline Are you aware of pharmacovigilance Programme of India? & $44(24.72 \%)$ & $138(79.77 \%)^{* *}$ \\
\hline Can nurses report ADRs? & $107(60.11 \%)$ & $162(93.64 \%)^{* *}$ \\
\hline \multicolumn{3}{|l|}{ Attitude of nurses toward ADR reporting } \\
\hline ADRs of old drugs should be reported & $78(43.82 \%)$ & $100(57.8 \%)^{*}$ \\
\hline ADRs of new drugs alone should be reported & $68(38.2 \%)$ & $34(19.65 \%)^{* *}$ \\
\hline Only serious life-threatening ADRs should be reported & $126(70.79 \%)$ & $5(2.89 \%)^{* *}$ \\
\hline ADRs of blood and components should be reported & $84(47.19 \%)$ & $110(63.58 \%)^{*}$ \\
\hline ADRs of vaccines should be reported & $80(44.94 \%)$ & $111(64.16 \%)^{* *}$ \\
\hline \multicolumn{3}{|l|}{ Practice of ADR reporting of nurses } \\
\hline ADRs were observed during duty & $131(73.6 \%)$ & $146(84.39 \%)^{*}$ \\
\hline Doctor on duty informed about ADR & $121(67.98 \%)$ & $138(79.77 \%)^{*}$ \\
\hline
\end{tabular}

$\mathrm{p}<0.05$ was considered statistically significant. ${ }^{*} \mathrm{p}<0.05,{ }^{* *} \mathrm{p}<0.01$ : Post-IP as compared to pre-IP. ADR: Adverse drug reaction, Post-IP: Post-intervention phase,

Pre-IP: Pre-intervention phase, CHA: Civil Hospital Ahmedabad 
a significantly greater number of nurses in the Post-IP (155/173, $89.6 \%$ ) opined that training in pharmacovigilance should be included in the nursing syllabus than in the pre-IP $(139 / 178,78.1 \%)(\mathrm{P}<0.05)$. Attitude of nurses on the type of ADRs to be reported in both pre-IP and post-IP is listed in Table 1.

\section{Practice}

Three questions in the KAP questionnaire assessed the practice of nurses regarding ADR reporting. As compared to pre-IP, in post-IP, a significantly higher number of nurses reported of having encountered an ADR and informing the same to doctors $(\mathrm{P}<0.05)$ (Table 1$)$. Majority of nurses in both the phases mentioned that doctors are their source of information about ADRs (pre-IP 126/178; post-IP: 142/173). Practical experience was stated as a source of information by a greater number of nurses in pre-IP $(65 / 178)$ as compared to post-IP $(42 / 173)(\mathrm{P}<0.05)$. Other sources of information mentioned by nurses were textbooks (pre-IP 80/178; post-IP: 82/173) and colleagues (pre-IP 72/178; postIP: 80/173). Various difficulties in reporting ADRs were cited by nurses. The majority of respondents in pre-IP stated that they did not know how to report ADRs, which decreased significantly in post-IP $(\mathrm{P}<0.001)$. Majority nurses stated lack of time as hindrance in reporting ADRs in the post-IP (Fig. 1).

\section{Analysis of ADRs reported by the nurses}

A total of 39 ADRs were notified by the nurses during the study period. No ADR was reported during the pre-IP. In IP (10 months), 33 ADRs were reported. Thus, there was an increase in the number of ADRs reported during the IP compared to pre-IP. Only six ADRs were reported in the Post-IP (3 months), i.e., a statistically significant decrease in the number of ADRs reported was observed after the educational interventions were withdrawn. Thirty nurses (33 reports) in the IP and six nurses in the post-IP reported ADRs. The increase in the number of nurses reporting ADRs in IP and post-IP was significantly higher than in pre-IP $(\mathrm{P}<0.001)$. ADRs were notified personally to the investigator (19), dropped in drop boxes (12), and informed investigator telephonically (8). The causal association of the suspected drug, severity assessments, and preventability analysis is presented in Table 2. The majority of ADRs reported (23 of 39) were due to antimicrobials. Other causal drugs included iron preparations, vaccines, analgesics, blood products, vitamins, antiepileptic, and immunoglobulins. The most common symptoms of ADRs reported were itching, followed by rash, rigor, chills, diarrhea, nausea, shivering, fever, SJ syndrome, convulsion, vomiting, headache, abdominal cramps, palpitations, induration at the injection site, and numbness.

\section{Completeness of information in ADR notification forms}

All ADR notification forms received from the nurses were evaluated for completeness on a scale of $0-30$. The mean score of completeness of notification forms was $19.36 \pm 2.8$ in IP while it was $15.33 \pm 1.6$ in postIP $(\mathrm{P}<0.001)$

\section{Impact of interventions on the number of ADRs reported}

The maximum number of ADRs (10) was reported by nurses in the month following the workshop. A spurt in ADR reporting was observed after each SMS. In the post-IP, a gradual decrease in the number of ADRs reporting was observed. The relationship of ADR reporting with personal briefings and posters could not be evaluated due to logistic issues. Interestingly, the number of reports also dipped during the 2 months period when these interventions (except poster) could not be sustained due to logistic reasons (Fig. 2).

Qualitative analysis of the responses of nurses during personal briefings

During interaction with nurses in the personal briefing, most nurses (73) perceived time as the major constraint in reporting ADRs, saying "filling up of forms requires a long time," "we have many other primary responsibilities," and "we lack time to report." Nurses also opined that "ADRs occur rarely" (44), "we do not encounter ADRs" (58), and "we cannot detect/recognize ADRs" (42). Some nurses expressed reservations about reporting saying "it is not the responsibility

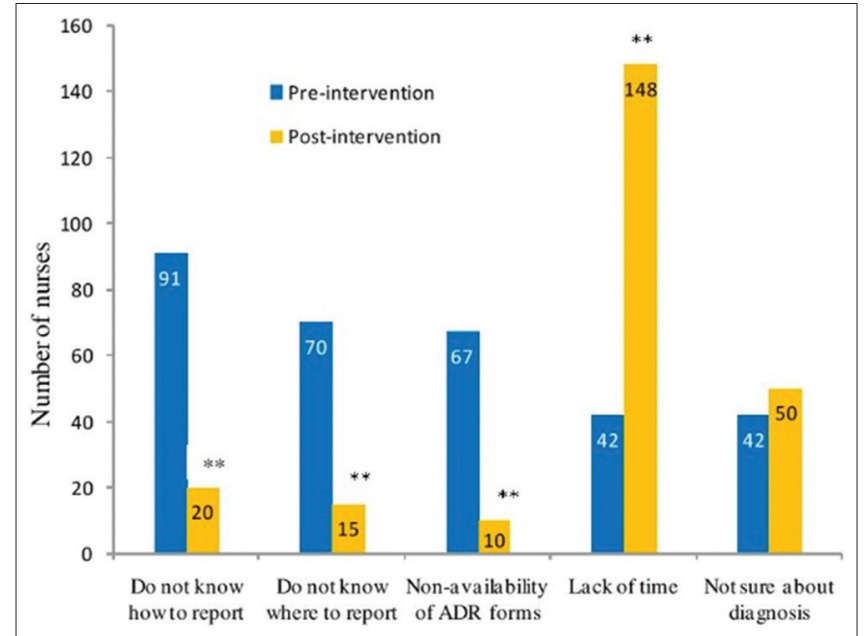

Fig. 1: Difficulties perceived by nurses in reporting adverse drug reactions in Civil Hospital Ahmedabad. $\mathrm{P}<0.05$ was considered statistically significant. ${ }^{* *} \mathbf{p}<0.001$ : Post-intervention phase as compared to pre-intervention phase

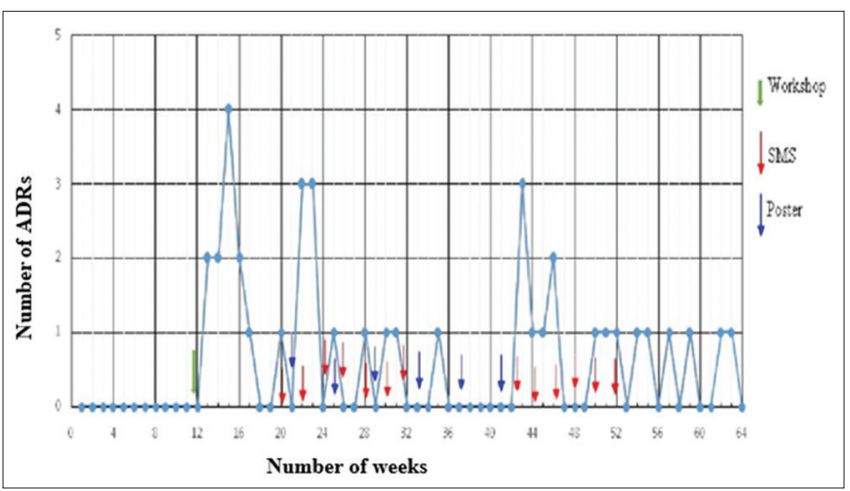

Fig. 2: Impact of various educational interventions on adverse drug reaction reporting by nurses: A correlation between time of interventions and the number of reports $(n=195)$

Table 2: Analysis of ADRs reported by nurses

\begin{tabular}{ll}
\hline & Number of cases (\%) \\
\hline Most common clinical presentation & $12(30.77 \%)$ \\
Itching & $11(28.21 \%)$ \\
Rash & $7(17.95 \%)$ \\
Rigor & $4(10.26 \%)$ \\
Chill & \\
Most common causal drug & $23(58.97 \%)$ \\
Antimicrobials & $5(12.82 \%)$ \\
Iron & $2(5.13 \%)$ \\
Analgesics & $20(51.28 \%)$ \\
Causality of suspected drug & $19(48.72 \%)$ \\
Possible & \\
Probable & $30(76.92 \%)$ \\
Preventability & $9(23.08)$ \\
Definitely preventable & \\
Not preventable & $15(38.46 \%)$ \\
Severity & $24(61.54 \%)$ \\
Mild & $0(0 \%)$ \\
Moderate &
\end{tabular}

Causality assessment by WHO-UMC scale; Severity analysis by Hartwig and Siegel scale and preventability analysis by modified Schumock and Thornton criteria. ADR: Adverse drug reaction

of nurses" (14), "we do not have confidence to report ADRs" (18), "reporting should be done by doctors" (17), "doctors may not like their 
reporting" (15), and "we fear legal liability" (20). Ten nurses opined that more training in this regard is necessary.

\section{DISCUSSION}

The PvPI encourages all health professionals, including nurses to report ADRs. However, an assessment made by PvPI shows that $64.66 \%$ of individual case safety reports were reported by clinicians, $14.75 \%$ by pharmacists, whereas other health-care professionals, including nurses and dentists, reported $18.83 \%$ of total ADRs annually [12]. Considering this low reporting rate of ADRs by nurses, this study was designed to evaluate the effect of certain educational interventions on KAP of ADR reporting by nurses.

In the present study, the response rate to KAP questionnaire was good in both pre- and post-intervention phases, albeit significantly higher in post-IP. A study at CHA to evaluate the KAP of ADR reporting of the 436 prescribers, showed a lower response rate of $61 \%$ [13]. In a study conducted in India among all health professionals, the response rate of nurses to the KAP questionnaire increased from $66.67 \%$ to $86.67 \%$ after interventions [14]. A higher response rate in the post-intervention phase in this study suggests a positive impact of these interventions. A KAP questionnaire, while being exploratory, is also a first step toward sensitizing health professionals about ADR reporting.

Before the educational interventions, participants in our study had poor knowledge about ADR and pharmacovigilance. A significant improvement in the knowledge score of the nurses was observed after educational interventions. A study carried out by Hajebi et al. among 250 nurses at Taleghani Hospital, Tehran, Iran, in 2005, found that nurses having adequate knowledge of pharmacovigilance increased from $24 \%$ to $42 \%$ after interventions [15]. A study carried out among 65 nurses in Government General Hospital, Guntur, Andhra Pradesh showed that the scores of knowledge (pre-intervention:11.8 \pm 3.70 and post-intervention $52.5 \pm 4.32$ ), attitude (pre-intervention: $29.8 \pm 7.94$ and post-intervention $47.6 \pm 9.81$ ), and practice (pre-intervention: $20 \pm 9.88$ and post-intervention $32.2 \pm 11.72$ ) of the nurses increased significantly after intervention [16]. Another study conducted in health professionals in Nepal over a period of 8 months showed a significant improvement in knowledge scores after educational interventions such as a lecture on pharmacovigilance and ADR reporting and poster session on pharmacovigilance [17]. Divergent to the results of these studies, a study carried out in the Philippines to assess the KAP of health professionals about ADR reporting revealed that nurses (86\%) were the most knowledgeable health professional regarding ADRs as compared to doctors (72\%) and pharmacists (61\%) [18]. This suggests that while the educational interventions do have an impact on ADR reporting, the extent of impact varies in different regions and in different health-care professionals. Hence, each country/region must decide and customize the educational interventions that are most suitable to their population of health professionals in their respective environments.

The results of our study show that few nurses were aware of the term "pharmacovigilance" before the educational interventions. In a study conducted by Radhakrishnan et al., in India among all health professionals, a lesser percentage of nurses $(55.2 \%)$ were aware of the definition of pharmacovigilance before intervention than in our study (63.48\%). However, the number of nurses knowing the meaning of "pharmacovigilance" increased after intervention in both [19]. Another Indian study conducted to evaluate the KAP of ADR reporting in health professionals also showed that the majority of the nurses (62.40\%) were aware of the correct meaning of "pharmacovigilance" which further improved after interventions [20]. Interestingly, few participants in the present study were aware of the ADR reporting system of CHA and PvPI before the intervention. In a similar study conducted in Bengaluru on health professionals, only $27 \%$ nurses were aware of the ADR reporting system of their hospital [21]. Contrary to the findings of our study, most of the nurses (73\%) were aware of the PvPI in a study conducted by Rehan et al. in Delhi on health professionals [22]. Thus, educational interventions were necessary to sensitize the nurses about the existing reporting system at CHA. Most nurses in this study were not aware that nurses, too, can report ADRs, but this ignorance was minimized following the educational interventions.

A positive attitude of the nurses at CHA toward ADR reporting was observed in this study. This further improved after educational interventions. The study carried out in Iran in 300 nurses showed an improvement in the attitude of nurses after educational interventions through a $3 \mathrm{~h}$ presentation on pharmacovigilance and ADR reporting [23]. Contrary to the results of these studies, one study carried out in the Philippines to assess the KAP of health professionals about ADR reporting showed that very few (27\%) nurses had a favorable attitude toward ADR reporting [18]. Studies conducted in India and Turkey also suggests that nurses exhibit a positive attitude toward ADR reporting [19,24]. A majority of nurses in this study opined that nurses should report ADRs. Contrary to this, in a study conducted in UAE, only 40.7\% nurses opined that nurses should report ADRs [25]. Before the interventions, the nurses in our study opined that only serious ADRs must be reported. This misbelief however was corrected after the educational interventions. A similar opinion about reporting only serious and life-threatening ADRs was expressed in another study conducted by Goka et al. before interventions which were modified positively after the intervention [16]. Majority respondents in this study stated that training in pharmacovigilance should be included in the syllabus for nursing students. Similar results were found in another study carried out in Central India, in which most nurses stated that pharmacovigilance should be taught to all health-care professionals [26]. A favorable attitude toward ADR reporting and a willingness to learn implies the positive impact of educational interventions.

Most respondents in our study stated that they had encountered an ADR during their duty. In another study from Guntur, India very few respondents (35.38\%) stated that they encountered ADRs [16]. The number of nurses who recognized ADRs increased after the interventions in both the studies. This increase may be attributed to an increased awareness of nurses toward the occurrence of ADRs after interventions. Nurses in this study reported certain difficulties in reporting ADRs. Some of these perceived difficulties were minimized after educational interventions. However, the concern for lack of time persisted in both phases, thereby implying that it was a logistic issue, rather than a genuine lack of awareness or ignorance. Lack of time has also been reported by most (83.95\%) nurses as a hindrance to reporting in a study carried out among 392 health professionals in Central India [26]. Most of the nurses in this study stated doctors are an important source of information about ADRs. This finding is useful and suggests that doctors can be a vital resource in educating nurses about ADRs. Many opportunities to educate nurses about detecting and monitoring ADRs exist for doctors, which can be reinforced further to improve ADR reporting by these health-care professionals.

Nurses began reporting ADRs during the intervention phase and reporting in the post-intervention phase, albeit at a lesser rate. The number of nurses reporting ADRs also increased after interventions. In a similar study conducted among 54 nurses in Sweden by Bäckström et al., the number of ADR reports submitted increased after interventions that comprised lectures on drugs and ADRs, ADR reporting, and special aspects of ADRs in elderly people. In a period of 12 months, 18 ADRs were reported by nurses of two geriatric departments in which interventions were carried out. However, only 15 reports were received from nurses of other 50 geriatric departments which did not receive any interventions [27]. A higher reporting rate after intervention in our study as compared to the study cited above may be due to the fact that multiple interventions were used in our study. In another study carried out in health professionals in India, including nurses, a fourfold increase in the number of reports was observed after interventions through interactive discussions, posters, and feedback letters [28]. This suggests that nurses can play an important role in detecting and reporting ADRs and educational interventions have a 
positive influence on ADR reporting. However, the sharp decline in the number of ADRs reported after withdrawing interventions reflects that the impact of interventions was not sustained and suggests the need for implementing these educational interventions on a continuous longterm basis. Studies conducted in other parts of the world on other health professionals also showed attenuation of the effects of educational interventions with time. In a study conducted in Spain, the difference in reporting rate between control and intervention groups lasted for 12 months [29]. In our study, the number of nurses reporting ADRs also increased after interventions. A study conducted in Karnataka also showed that a larger number of nurses ( 84 in pre-IP vs. 111 post-IP of a total of 124 nurses) reported ADRs after interventions [30].

During the IP, all interventions except posters had to be stopped for 2 months due to logistic reasons. An abrupt decrease in the number of ADRs was observed during this period. An increase in the rate of reporting after resuming these interventions was observed. This suggests that workshops, personal briefings, and SMSes have been more useful than posters in this regard. SMSes served as a reminder for nurses to report ADRs. Personal briefings had been proved to be very useful. This could be due to the fact that besides reminding and reinforcing the need of ADR reporting, various queries of the nurses were also discussed during these briefings. This implies that selection of educational interventions must be made based on the need, their efficacy, and impact in the selected population. Furthermore, a mix of different types of interventions is suggested to suit the varying needs of the population.

Most common ADRs reported by nurses in our study were cutaneous reactions and most common drug associated with ADRs is antimicrobials. This finding is similar to another study carried out in India by Belhekar and Munshi, among health professionals. In this study, of 189 ADRs reported in 1 year, 149 (76.2\%) were cutaneous reactions and $95(50.3 \%)$ were due to antimicrobials [28]. Frequent prescription of antimicrobials in India and increased adverse effects with the use of these drugs may explain these findings. While cutaneous ADRs are a common form of ADR [31], it is also a fact that they are usually easier to detect by an untrained person than other types of ADRs. This may also explain this finding of our study.

Due to the completeness of the ADR forms, severity analysis of the reports was possible in this study. In contrast to our study, in another study from Sweden, of 23 ADRs reported by nurses, 17 were serious [32]. While serious ADRs have been reported by prescribers, the fact that none of the nurses reported serious ADRs needs further evaluation. The quality of ADR reports submitted is also important for the success of a pharmacovigilance program. An ADR report must necessarily contain certain information to be meaningful and useful for causality analysis. The high score of the nurses regarding the completeness of ADR notification form can be attributed to various interventions like a hands-on exercise in the workshops on filling up these forms and addressing the queries of the nurses regarding filling up of the forms during personal briefings. These could explain the good quality of reports submitted by nurses in the intervention and post-IP. Results of our study were in concurrence with another study from Sweden, where nurses submitted high-quality ADR reports after receiving intervention through ADR information letters on three occasions, although the quantity of reports did not increase [33]. In a study conducted in 177 nurses in the UK to assess the role of community nurses in ADR reporting, the ADR reports submitted by nurses were found to be of the same quality as those of physicians [34]. In our study, the quality of reports was found to be poorer after the interventions were withdrawn. Thus, continuous training for a longer time is suggested to have a sustainable impact on the quality of reporting. This may be particularly important when initiating the practice of ADR reporting in a population of health-care professional.

\section{CONCLUSIONS}

The study demonstrated a positive impact of educational interventions on KAP of ADR reporting. However, sustainability of the impact of these interventions is a major concern as proven by a decrease in reporting rate after the withdrawal of interventions. This suggests a need for continuous and long-term educational interventions. The study recommends extending educational interventions to other health professionals and stakeholders such as pharmacists and consumers and implementing the educational interventions for a longer duration for improved reporting of ADRs.

\section{ACKNOWLEDGMENTS}

The authors acknowledge the Superintendent of the Hospital, Dr. M. M. Prabhakar and the Nursing Superintendent Mr. B.K. Prajapati, for the permissions for conducting and guidance during the study.

\section{AUTHORS' CONTRIBUTIONS}

Dr. Supriya has written this manuscript. Dr. Chetna and Dr. Jigar were involved in reviewing, editing, and guiding to finalize the manuscript.

\section{CONFLICTS OF INTEREST}

There are no conflicts of interest.

\section{AUTHORS' FUNDING}

This article is self-funded.

\section{REFERENCES}

1. Wester K, Jonsson AK, Spigset O, Druid H, Hagg S. Incidence of fatal adverse drug reactions: A population based study. Br J Clin Pharmacol 2008;65:573-9.

2. Davies EC, Green C, Taylor S, Williamson PR, Mottram D, Pirmohamed M. Adverse drug reactions in hospital in-patients: A prospective analysis of 3695 patient-episodes. PLoS One 2009; 4:e4439.

3. Sriram S, Ghasemi A, Ramasamy R. Prevalence of adverse drug reactions at a private tertiary care hospital in South India. J Res Med Sci 2011;16:16-25.

4. Raut A, Diwan A, Patel C, Patel P, Pawar A. Incidence, severity and financial burden associated with adverse drug reactions in medicine inpatients. Asian J Pharm Clin Res 2011;4:103-11.

5. Oshikoya KA, Awobusuyi JO. Perceptions of doctors to adverse drug reaction reporting in a teaching hospital in Lagos, Nigeria. BMC Clin Pharmacol 2009;9:14.

6. Yadav S. Status of adverse drug reaction monitoring and pharmacovigilance in selected countries. Indian $\mathrm{J}$ Pharmacol 2008;40:S4-9.

7. Kalaiselvan V, Prasad T, Bisht A, Singh S, Singh GN. Adverse drug reactions reporting culture in Pharmacovigilance Programme of India. Indian J Med Res 2014;140:563-4.

8. Hanafi S, Torkamandi H, Hayatshahi A, Gholami K, Shahmirzadi NA, Javadi MR. An educational intervention to improve nurses' knowledge, attitude, and practice toward reporting of adverse drug reactions. Iran J Nurs Midwifery Res 2014;9:101-6.

9. Pedrós C, Vallano A, Cereza G, Mendoza-Aran G, Agustí A, Aguilera C, et al. An intervention to improve spontaneous adverse drug reaction reporting by hospital physicians: A time series analysis in Spain. Drug Saf 2009;32:77-83.

10. Herdeiro MT, Ribeiro VI, Ferreira M, Polónia J, Falcão A, Figueiras A. Workshop-and telephone-based interventions to improve adverse drug reaction reporting: A cluster-randomized trial in Portugal. Drug Saf 2012;35:655-65.

11. Patel SV, Desai CK, Patel PP, Dikshit RK, Patel. An impact of educational interventions on reporting of adverse drug reactions. Int $\mathrm{J}$ Pharm 2015;5:485-92.

12. Thota P, Kalaiselvan V, Singh GN. Current status and challenges of pharmacovigilance programme of India. In: $2^{\text {nd }}$ International Conference and Exhibition on Pharmacovigilance and Clinical Trials. Texas, USA: Hilton San Antonio Airport; 2013.

13. Desai CK, Iyer G, Panchal J, Shah S, Dikshit RK. An evaluation of knowledge, attitude, and practice of adverse drug reaction reporting among prescribers at a tertiary care hospital. Perspect Clin Res 2011;2:129-36

14. Palanisamy S, Kumaran AK, Rajasekaran A. A study on assessment of knowledge about adverse drug reactions. Pharm Lettr 2013;5:41-52. 
15. Hajebi G, Mortazavi SA, Salamzadeh J, Zian A. A survey of knowledge, attitude and practice of nurses towards pharamacovigilance in Taleqani hospital. Iran J Pharm Res 2010;9:199-206.

16. Goka PK, Nadendla S, Gangisetty S, RamaRao NV, Nadendla RR. An evaluation of impact of educational intervention on pharmacovigilance and ADR reporting among nurses in tertiary care teaching hospital. World J Pharm Res 2015;4:2568-75.

17. Jha N, Rathore DS, Shankar PR, Gyawali S, Alshakka M, Bhandary S. An educational intervention's effect on healthcare professionals' attitudes towards pharmacovigilance. Australas Med J 2014;7:478-89.

18. Carandang RR, Cao K, Jose NB, Almonte FD, Tinio RM. Knowledge and attitudes on adverse drug reaction reporting of selected hospitalbased health practitioners in Manila, Philippines. Sch Acad J Pharm 2015;4:301-7.

19. Radhakrishnan R, Vidyasagar S, Varma DM. An educational intervention to assess knowledge attitude practice of pharmacovigilance among health care professionals in an Indian tertiary care teaching hospital. Int J Pharm Tech Res 2011;3:678-92.

20. Gupta SK, Nayak RP, Shivaranjani R, Vidyarthi SK. A questionnaire study on the knowledge, attitude, and the practice of pharmacovigilance among the healthcare professionals in a teaching hospital in South India. Perspect Clin Res 2015;6:45-52.

21. Muraraiah S, Rajarathna K, Sreedhar D, Basavalingu D, Jayanthi CR. A questionnaire study to assess the knowledge, attitude and practice of pharmacovigilance in a paediatric tertiary care centre. J Chem Pharm Res 2011;3:416-22.

22. Rehan HS, Sah RK, Chopra D. Comparison of knowledge, attitude and practices of resident doctors and nurses on adverse drug reaction monitoring and reporting in a tertiary care hospital. Indian J Pharmacol 2012;44:699-703.

23. Hanafi S, Torkamandi H, Hayatshahi A, Gholami K, Javadi M. Knowledge, attitudes and practice of nurse regarding adverse drug reaction reporting. Iran J Nurs Midwifery Res 2012;7:21-5.

24. Vural F, Ciftci S, Vural B. The knowledge, attitude and behaviours of nurses about pharmacovigilance, adverse drug reaction and adverse event reporting in a state hospital. North Clin Istanb 2014;1:147-52.

25. John LJ, Arifulla M, Cheriathu JJ, Sreedharan J. Reporting of adverse drug reactions: An exploratory study among nurses in a teaching hospital, Ajman, United Arab Emirates. Daru 2012;20:44.

26. Torwane NA, Hongal S, Gouraha A, Saxena E, Chavan K. Awareness related to reporting of adverse drug reactions among health caregivers: A cross-sectional questionnaire survey. J Natl Accredit Board Hosp Healthc Prov 2015;2:23-9.

27. Bäckström M, Mjörndal T, Dahlqvist R. Spontaneous reporting of adverse drug reactions by nurses. Pharmacoepidemiol Drug Saf 2002;11:647-50.

28. Belhekar MN, Munshi R. Effect of educational interventions in improving spontaneous adverse drug reaction reporting in a tertiary care setting. Asian J Pharma Health Sci 2014;4:903-8.

29. Figueiras A, Herdeiro MT, Polónia J, Gestal-Otero JJ. An educational intervention to improve physician reporting of adverse drug reactions: A cluster-randomized controlled trial. JAMA 2006;296:1086-93.

30. Ganachari MS, Patil PA, Soham S, Nidhi Z. Impact of educational interventions on adverse drug reaction reporting. Indian J Pharm Pract 2011;4:26.

31. Farshchian M, Ansar A, Zamanian A, Ghasem RR, Arash KA, Mehdi F. Drug-induced skin reactions: A 2-year study. Clin Cosmet Investig Dermatol 2015;8:53-6.

32. Bäckström M, Ekman E, Mjörndal T. Adverse drug reaction reporting by nurses in Sweden. Eur J Clin Pharmacol 2007;63:613-8.

33. Johansson ML, Hägg S, Wallerstedt SM. Impact of information letters on the reporting rate of adverse drug reactions and the quality of the reports: A randomized controlled study. BMC Clin Pharmacol 2011;11:14.

34. Morrison-Griffiths S, Walley TJ, Park BK, Breckenridge AM, Pirmohamed M. Reporting of adverse drug reactions by nurses. Lancet 2003;361:1347-48 